\title{
From the Editors-in-Chief
}

\section{International strategy in the struggle against gastric cancer}

\section{OICHIRO KOBORI}

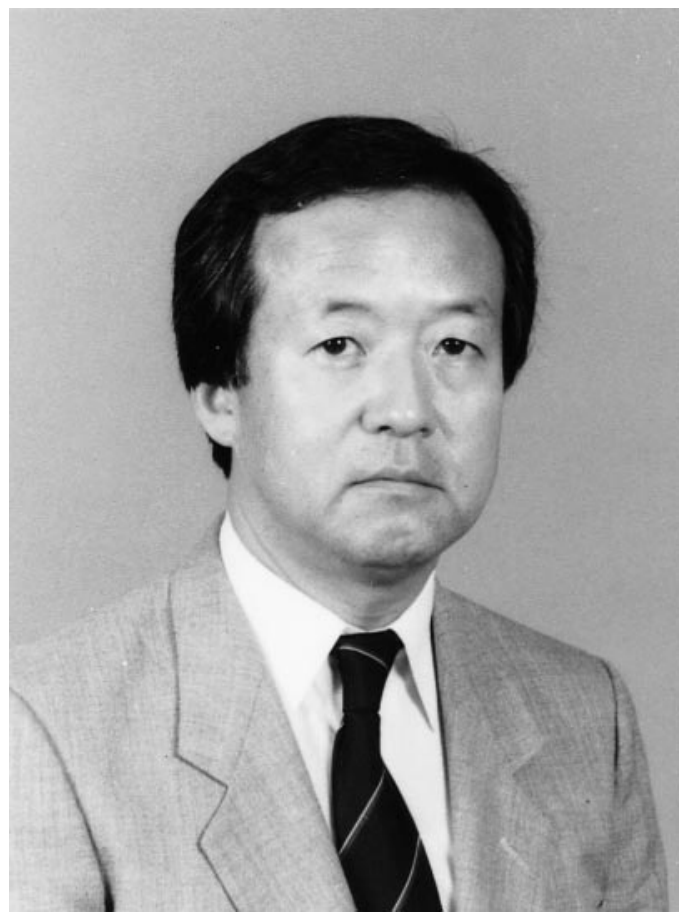

Oichiro Kobori

Vice-Director, International Medical Center of Japan
Gastric cancer has long been the leading cause of death from cancer in Japan, a fact which motivated the establishment of various measures for the solution of this national problem. Firstly, in 1962, the Japanese Research Society for Gastric Cancer was founded and General Rules for the Treatment of Gastric Cancer were issued, promoting to a remarkable degree the standardization of both the diagnosis and treatment of this disease in Japan. Secondly, in the following year, general registration of all gastric cancer cases became standard practice and the total number of registered cases reached 264572 by 1990 . The data gathered proved particularly profitable for comparisons between results of individual hospitals and general treatment, making possible objective judgment for surgical planning in each clinical department.

The most fruitful result of the first period of the above national project in the past 35 years disclosed the fact that the proportion of early cancer increased: from $10.3 \%$ between 1963 and 1967 to $42.7 \%$ in the period between 1988 and 1990, and that the second period revealed remarkable progress in the basic study of gastric cancer: epidemiology, natural history, biochemistry, molecular pathology, genetics and experimental carcinogenesis. This progress was combined with that of recent technology including that of medical industries and enabled the establishment of the patient-oriented treatment principle for gastric cancer ranging from endoscopic mucosectomy, minimal invasive surgery to super-extended surgery combined with chemotherapy.

If something seemed lacking to the stream of the Japanese Anti-Gastric Cancer Campaign during these 35 years, it was, perhaps, the philosophy of the Almaata Declaration (1978): "Health for all by the year 2000." What should be stressed is that this does not mean the Japanese Gastric Cancer Association (JGCA) was reluctant to share their own experiences on gastric cancer with surrounding countries. Study conferences were held by the Japanese International Cooperation 
Agency (JICA) 28 times since 1969, and the total number of participants from 59 countries amounted to 463 . The problem seems to be that these international activities were always carried out in the framework of the Japanese Anti-Gastric Cancer Campaign under limited geographical, financial conditions and mentality.

In Western countries the incidence of gastric cancer has been decreasing during the past half-century. The screening system which was so profitable for early detection of gastric cancer in Japan is not relevant today in other countries where most of the patients are over 70 , obese, with cardiovascular disorders, which lead to the higher incidence of severe postoperative complications and operative deaths. These facts do not encourage extended lymphnode dissection. Rather, chemotherapy would be the preferential choice. In the second International Gastric Cancer Congress which took place in Munich in 1997, the main discussion was concentrated on the International Documentation System (IDS), or how we can establish the international description of gastric cancer. At present, UICC (TNM) Classification and General Rules of Gastric Cancer of Japan are broadly accepted as the description of gastric cancer. The former, however, was mainly used for stage classification and the latter as a guideline for operative treatment of gastric cancer. Moreover, the latest UICC Classification, issued in 1997, determined cancer stages according to actual numbers of metastatic lymphnodes; the reciprocal adjustment of both classifications became impossible. This fact apparently increases the impor- tance of IDS as the common basic formulation looking towards future international clinical research of gastric cancer.

In developing countries most gastric cancer patients come to hospitals at an advanced stage and therefore detection of early gastric cancer is rare, and the double contrast method in X-ray diagnosis and endoscopic techniques are at present difficult to develop. Another characteristic of gastric cancer patients in developing countries is that postoperative patients seldom come to hospital again; the surgical staff has no opportunity to ascertain the course and results of their own surgical treatment which diminishes motivation for systematic lymphnode dissection aiming at the betterment of the prognosis.

While conditions vary from country to country, the reality is that only several groups of young surgeons in England, Holland, Hong Kong and Vietnam are attempting gastrectomy with lymphnode dissection which is considered standard in Japan.

The International and Japanese Gastric Cancer Associations were founded in 1995 and 1998 respectively. Gastric cancer is no longer a geographically limited disease treated in limited ways and in limited areas. Its treatment should be based on international consent and I hope this journal will henceforth provide a forum where various ideas may be exchanged until a consensus is arrived at.

Oichiro Kobori, Editor-in-Chief 